\title{
ETS Transcription Factors in the Tumor Microenvironment
}

\author{
Fu Li ${ }^{1,2}$, Julie A. Wallace ${ }^{1,3}$ and Michael C. Ostrowski ${ }^{*}{ }^{1,2}$ \\ ${ }^{I}$ Department of Molecular and Cellular Biochemistry, College of Medicine, ${ }^{2}$ Department of Molecular Genetics, \\ ${ }^{3}$ Molecular, Cellular and Developmental Biology Program, The Ohio State University, Columbus, OH 43210, USA
}

\begin{abstract}
ETS factors are involved in cancer progression through transcriptional regulation of factors mediating cell cycle, cell growth, apoptosis, cell adhesion and migration. The biological processes regulated by ETS factors are important not only in tumor cells, but also in the surrounding cells comprising the tumor microenvironment. Additionally, ETS factors can serve as transcriptional activators and repressors to regulate gene expression in a cell context-specific fashion. Here we discuss recent advances in which the regulatory roles of ETS factors in stromal cells are critical during both development and cancer. These new findings uncover the importance of ETS signaling in the stromal compartment of tumors and shed light on new potential mechanisms of ETS factors.
\end{abstract}

Keywords: ETS factors, tumor microenvironment, angiogenesis.

\section{INTRODUCTION}

Since their discovery almost three decades ago $[1,2]$, E26 transcription specific sequence (ETS) transcription factors have been implicated in many types of cancer. Currently, 27 ETS factors have been identified in human and 26 in mouse [3]. Initial in vitro studies revealed the ability of ETS factors Ets 1 and Ets2 to cause transformation when overexpressed [4]. Later etiological studies found expression levels of ETS factors to be correlated with tumor progression in several types of cancer, ranging from thyroid and liver cancer to lung and breast cancer [5]. The mechanisms responsible for the upregulation of these factors can include gene amplification, mutation, translocation and gene fusion [6]. At the molecular level, ETS factors can serve as transcriptional activators or repressors of genes regulating cell cycle, apoptosis, cell migration and extracellular matrix remodeling, all of which play key roles during cancer progression [5]. The involvement of ETS factors in cancer has been summarized in several excellent reviews [5, 7]. Here, we highlight the cell non-autonomous roles of ETS factors from within various stromal cellular compartments during development and during tumor progression and metastasis. In particular, we focus on studies that use genetic approaches to uncover the in vivo functions of ETS factors in stromal cells.

\section{STROMAL ACTION OF ETS FACTORS IN DEVE- LOPMENT}

In order to evolve into tumors, cells have to become independent of and insensitive to growth signals, escape apoptosis, avoid differentiation signals, recruit blood vessels, and break through basement membranes to invade tissue and metastasize [8]. These critical steps of cancer progression

*Address correspondence to this author at the Department of Molecular \& Cellular Biochemistry, The Ohio State University, 810 Biomedical Research Tower, 460 West 12th Avenue, Columbus, OH 43210, USA; Tel: 614-6883824; Fax: 614-688-4181; E-mail: michael.ostrowski@osumc.edu mimic several stages of embryonic development. Indeed, tumorigenesis and organogenesis may utilize many of the same genes and pathways [9]. ETS factors are involved in several processes in which their stromal function can affect development. For example, the function of ETS factors in directing the formation of a complex, branched vascular system is critical for organ development. A second example is the role of ETS-factors in regulating sonic hedgehog (SHH) expression during limb development $[10,11]$.

\subsection{ETS Function In Endothelial Cells During Develop- ment}

The vascular system is formed through the processes of vasculogenesis and angiogenesis during embryonic development. Vasculogenesis is the process by which endothelial cells form primitive blood vessels, while angiogenesis involves the branching and remodeling of these primitive vessels to form the mature vascular system [12]. ETS factors have been shown to be expressed in angioblasts, the mesodermally-derived precursors of endothelial cells, and recent reviews have detailed ETS factor function in endothelial cell proliferation, migration and survival necessary for blood vessel development $[13,14]$.

Computational analysis identified 31 ETS-domain containing genes in the Zebrafish genome. Twelve of these genes are expressed in hematopoietic and (or) endothelial cells during zebrafish embryogenesis (ets 1, ets 2, etsrp, fli1, flilb, erg, fev, gabpa, elf1, elf2, pu.1, and elk4) [15]. Studies using the zebrafish model system have been particularly informative in uncovering the complex role ETS factors play in formation of the vasculature in vertebrates. For example, one study identified four zebrafish ETS-factors expressed in endothelial cells at early stages of development: etsrp (a zebrafish ETS factor for which there is no mammalian orthologue, but most closely related to ETS1 and ETS2), fli1, $f l i l b$, and ets 1 . Morpholino knockdown of any one of these four factors alone resulted in a partial endothelial phenotype, with etsrp knockdown yielding the most severe phenotype. In contrast, knockdown of all four factors simultaneously 
resulted in near complete loss of endothelial cells and blood vessel formation [16]. In a similar study by another group of investigators, three ETS factors expressed in angioblasts, etsrp, flil and erg were knocked down using a morpholino strategy [15]. Similar results were obtained, with etsrp knockdown resulting in the most severe phenotype; however, knockdown of flil and erg together resulted in apparent defects in angiogenesis without affecting vasculogenesis.

In mouse genetic studies, knockout of Etv2 (Er7l) resulted in a significant decrease in endothelial cells and defective vasculogenesis, while overexpression resulted in increased blood vessel formation, indicating Etv2 may be a functional orthologue of etsrp required for vasculogenesis $[17,18]$. In addition, Etv2 was also found to collaborate with the forkhead factor FoxC2 to directly regulate expression of a set of genes important for endothelial cell differentiation, including transcription factor Mef2c [19]. However, Etv2 is not expressed beyond E10.5 during mouse development, suggesting that it is not required for angiogenesis $[17,20]$.

Genetic analysis of additional mouse orthologues of zebrafish genes implicated in blood vessel development failed to demonstrate strong vascular phenotypes. Mouse knockouts of Ets 1 and Erg revealed no obvious vascular or endothelial cell defects [21-23], and while Fli1 mutants were shown to have leaky blood vessels, no obvious defects in vasculogenesis or angiogenesis were observed [24, 25]. Additionally, compound heterozygotes of Flil and Erg mutations had no vascular phenotype [26].

Recent work from our lab demonstrated that Ets 1 and Ets2 act in a redundant and endothelial-cell autonomous fashion to promote embryonic angiogenesis during murine development [27]. Using both in vivo and in vitro Cre/loxP mediated knockout of Ets 1 and Ets 2 specifically in endothelial cells, we observed drastically reduced vascular branching and significantly increased apoptosis in endothelial cells. This increase in cell death could be explained by deregulation of $B c l-x L$ and $c I A P$, critical anti-apoptosis genes, both down-regulated in the double-knockout endothelial cells [27]. In addition, Ets 1/Ets 2 null endothelial cells expressed lower levels of genes encoding extracellular proteases like $M m p 9$, suggesting these factors also regulate genes necessary for endothelial cell migration, perhaps coordinating cell survival and migration during angiogenesis.

One major conclusion emerging from these studies is that ETS factor function is overlapping and redundant during vasculogenesis and angiogenesis. The exact nature of this overlapping or redundant function needs further clarification; that is, the target specificity of the ETS-factors remains an open and intriguing question. A second conclusion is that ETS factor function is temporally regulated; for example, with etsrp or Etv2 being more important at early stages of cell differentiation and blood vessel formation and Ets1/Ets2 at later stages. Whether this temporal regulation is mediated through combinations of ETS-factors via feed-forward/feedback loops is an interesting possibility deserving attention.

\subsection{ETS Function During Limb Formation}

Recent work demonstrated that the functions of Etv4/ $P e a 3$ and Etv5/Erm are required in a cell non-autonomous fashion for correct patterning of the limb during mouse development [10]. Two classes of paracrine factors mediating limb development are Sonic hedgehog (Shh) and fibroblast growth factors (FGFs) [reviewed in 10]. Shh is produced from the Zone of Polarizing Activity (ZPA), a region in the posterior mesenchyme of the limb bud. Shh is required for the development of the anterior-posterior (A-P, thumb to little finger) axis of the limb, and subsequently loss of Shh activity results in fewer digits while ecotopic expression results in more digits. FGFs from the apical ectodermal region (AER) are required for proper development of the limb on the proximal-distal (P-D, shoulder to finger tip) axis. FGFs are also critical for initiating and maintaining Shh activity in the ZPA, and Shh can indirectly affect FGF expression and activity.

Etv 4 and Etv 5 are expressed in the limb bud mesenchyme adjacent to the AER, and genetic inactivation of FGF signaling in this mesenchyme resulted in reduced expression of Etv $4 / 5$, indicating they may be downstream targets of FGF in P-D development. However, ablation of both genes in mesoderm early in development led to unexpected defects in A-P development, resulting in development of extra digits. This phenotype correlated with overexpression of Shh in the ZPA as well as ectopic expression outside this region. Thus, Etv4/5 act to repress Shh signaling in an FGF dependent fashion, restricting ectopic Shh expression, but also modulating the level of expression within the ZPA [10].

Whether Etv $4 / 5$ directly regulate $S h h$, or whether the effect is indirect, has not been directly determined. However, expression of a number of transcription factors known to regulate Shh during limb development, including Handl, Twist 1 , and Gli3, were not affected in the Etv $4 / 5$ null mesenchyme, suggesting the ETS-factor effect might be direct [10]. Interestingly, overexpression of a dominant negative (DN) form of Etv4 in the developing limb mesenchyme affects both A-P and P-D limb development [11]. Given that Ets-DN genes generally are not specific and generically block ETS-factor function, these results suggest ETS-factors may be acting as activators and repressors during limb development.

Another indirect line of evidence suggesting a role for ETS-factors in Shh regulation and limb development arises from defining the regulatory element responsible for control of Shh expression in the ZPA. Genetic studies in mouse first identified mutations in a long range cis-regulatory sequence located $1 \mathrm{MB}$ distal to the $S h h$ gene that resulted in extra digit phenotypes; similar mutations located on chromosome $7 \mathrm{q} 36$ could be found in humans with the pre-axial polydactyl extra digit phenotype [reviewed in 28]. This distal enhancer is conserved from zebrafish to human and at least 12 mutations resulting in pre-axial polydactyl have been identified. Inspection of this element revealed it to contain multiple conserved potential Ets core sites and at least one known mutation overlapped with a potential ETS-binding site. Since FGF signaling is required for activation of $S h h$ expression, it seems likely that ETS-factors contribute to both positive and negative regulation of Shh in the ZPA through this element.

From these observations, we hypothesize that a set of ETS-factors, including Etv $4 / 5$ and other yet to be identified family members, play overlapping and redundant roles in limb development in a fashion similar to ETS-factor function in blood vessel development. Additional genetic experiments 
will be required to test this hypothesis. If it is correct, comparison of ETS-family networks in these different developmental processes will help to further define how specificity in the large ETS-family is achieved.

\section{ETS FACTORS IN CANCER STROMA}

The various cellular components comprising cancer stroma include macrophages, endothelial cells and fibroblasts. In addition to these cells, a non-cellular compartment of the stroma is also present and is composed of extracelluar matrix (ECM), growth factors and cytokines. ETS factors are known to regulate expression of many of the non-cellular components of tumor stroma, such as uPA, MMPs, cytokines (e.g. TNF-a and FGF-10) and chemokines (e.g. IL-2) [29]. Therefore, it can be hypothesized that ETS factors are active regulators in tumor stroma.

Direct genetic evidence implicating ETS factors as important modulators in the stroma was provided by work from Robert Oshima's lab [30]. This group demonstrated that mammary tumor growth in the MMTV-PyMT mouse model was restricted in mice that were homozygous for the hypomorphic Ets $2^{A 72}$ allele, which encodes a protein product that is unable to be phosphorylated by Ras/ERK signaling. Tumors from wild-type mice transplanted into mammary glands of Ets $2^{A 72 / A 72}$ mice grew more slowly compared to tumors transplanted into mice expressing wild-type Ets2. Thus, the active state of Ets 2 was shown to impact growth of mammary tumors from stromal cells [30]. Analysis of tumors from $E t s 2^{A 72 / A 72}$ mice suggested that lower production of extracellular matrix proteases like MMP9 correlated with lower ETS2 activity [30]. In a follow up study, this group specifically knocked out Ets 2 in $P y M T$ mammary tumor cells using a loxP conditional allele of Ets 2 and MMTV-Cre, and found this deletion had no effect on tumor initiation [31]. In contrast, generalized conditional knockout of Ets 2 using Mox2-Cre (MORE) also resulted in lower tumor growth. Taken together, these studies support the stromal function of Ets2 in promoting mammary tumor growth. However, evidence implicating specific stromal cell type(s) in which Ets 2 function was required was lacking.

\subsection{The Pten/Ets2 Axis in Tumor Fibroblasts}

Fibroblasts are the most abundant stromal cell type in mammary tumors. These cells have a profound effect on tumorigenesis through regulation of ECM, epithelial cell differentiation, inflammation and angiogenesis [32]. For example, it was previously reported that fibroblast specific knockout of Tgf-br (transforming growth factor beta receptor) caused neoplasia in prostate and carcinoma in the forestomach of mice [33]. We have recently showed that disruption of Pten specifically in stromal fibroblasts, using a Cre driven by the Fibroblast Specific Protein promoter (FSPcre), in the context of the MMTV-ErbB2 mammary tumor model caused accelerated tumor growth, expanded ECM and increased macrophage infiltration [34]. Ets2 expression was upregulated in fibroblasts with Pten deletion, and the ETS2 protein was activated by T72-phosphorylation in both fibroblasts and epithelial cells. Deletion of both Ets2 and Pten in stromal fibroblasts resulted in dramatically reduced tumor growth, as well as decreased inflammation and angio- genesis, in comparison to Pten deletion alone. These results indicate that Pten can suppress mammary tumors from stromal fibroblasts, and that Ets 2 is an important target activated when Pten function is lost.

Using a similar strategy, our group inactivated an Ets2loxP allele with FSP-cre in the context of the well-characterized MMTV-PyMT mammary tumor model. Fibroblast specific Ets2 inactivation did not alter mammary gland development in mice without the $P y M T$ oncogene. However, deletion of Ets 2 in fibroblasts caused a significant reduction in tumor size in MMTV-PyMT animals (F.L. and M.C.O., manuscript in preparation). Pathological characterization showed that the absence of Ets 2 in fibroblasts caused reduced epithelial tumor cell proliferation and delayed tumor progression. We also confirmed the results of Oshima's group [31], finding that deletion of Ets2 in the mammary epithelial cells had no effect on tumor growth or progression (F.L. and M.C.O., manuscript in preparation).

Gene expression profiling of tumor fibroblasts with or without $E t s 2$ defined a tumor specific transcription program modulated by Ets 2 . This program included genes promoting angiogenesis and ECM remodeling, in particular extracellular proteases and protease inhibitors (F.L. and M.C.O., manuscript in preparation). The majority of these genes were downregulated when Ets 2 was deleted, and importantly, the majority of genes were deregulated in a tumor-specific fashion. One representative gene revealed by this analysis was matrix metalloprotease 9 (MMP9), which is involved in the turnover of gelatin, type IV collagen (basement membrane) and other components of the ECM. Degradation of ECM may facilitate cancer cell migration and metastasis [35]. MMP9 can also release growth factors such as $V G_{164}$ from binding with ECM molecules thus promoting angiogenesis [36]. Ets2 regulated $M m p 9$ gene expression directly, and disruption of Ets 2 in tumor fibroblasts reduced MMP9 gene expression that subsequently caused decreased protease activity in vivo, as measured by fluorescent in situ zymogen assays. Such transcriptional regulation appears not to be limited to mouse models, as demonstrated by a survey of protein levels of ETS2 and MMP9 in human tumor tissue arrays, which revealed a significant correlation between the two molecules. These data indicate ETS2 in tumor fibroblasts might be controling a program that promotes sufficient new blood vessel formation necessary for cancer cells to thrive.

In support of this hypothesis, loss of Ets2 in the PyMT model led to decreased localization of active $\mathrm{VEGF}_{164}$, reduced activation of VEGF receptor signaling in tumor endothelial cells, and overall decreased tumor angiogenesis [34]. Importantly, tumor fibroblasts expressing Ets 2 have the ability to stimulate angiogenesis in a matrigel plug assay much more efficiently than fibroblasts lacking Ets2 (F.L. and M.C.O., unpublished data).

Three conclusions can be drawn from these studies. First, the studies show that Ets2 regulates an oncogenic gene expression program in tumor stromal fibroblasts that indirectly promotes tumor growth. This role of Ets 2 is specific to the stroma since Ets2 is apparently dispensable for tumor progression in epithelial tumor cells. These data support the notion that in epithelial tumor cells, Ets2 function is redun- 
dant with other ETS-family members and/or collaborating transcription factors while its role in the tumor stroma is unique. Thus, Ets 2 represents an example of a class of genes with activities restricted to tumor stromal fibroblasts. The activation of an Ets 2 stromal fibroblast-specific expression program represents one mechanism by which the tumor coopts the microenvironment to serve in its progression to malignancy.

Secondly, the consequences of inactivating this Ets2driven stromal program remain tumor specific, sparing normal development of the mammary gland. Finally, the Ets2 mechanism involves the activation of a gene expression program that leads to remodeling of the ECM and increased angiogenesis. Targeting the tumor vasculature remains an attractive therapeutic target. However, current strategies in which single molecules such as VEGF are targeted have failed clinically. These results indicate that the changes in tumor angiogenesis observed in vivo are likely regulated by the cumulative action of many Ets2-target genes acting in concert. Defining and targeting the pathway hubs controlled by Ets 2 provides an alternative strategy that should be more effective at altering multiple cell-cell and cell-matrix interactions required for tumor angiogenesis.

\subsection{Ets2 in Tumor Associated Macrophages}

In addition to stromal fibroblasts, tumor associated macrophages (TAMs) have also been implicated in tumor progression [37-39]. Within the tumor stroma, distinct subpopulations of macrophages are speculated to individually contribute to tumor progression by mediating distinct processes such as angiogenesis, invasion, and immunosuppression [40]. PyMT animals carrying homozygous null mutations of CSF-1 (op), a well-known growth factor involved in macrophage survival, proliferation and differentiation, had no obvious defects in tumor initiation, incidence or tumor size. However, these $o p$ animals developed far less invasive and metastatic carcinoma than those carrying the PyMT transgene only [41].

CSF-1 signaling in macrophages has been shown to activate the MAPK pathway and downstream transcription factors, including Ets 1 and Ets2 [42]. To evaluate whether Ets2 is an important downstream factor of CSF1-R signaling in TAMs, genetic inactivation of Ets 2 was achieved using LysMCre transgenic mice. In these mice, Cre expression is under the control of the macrophage-specific lysozyme promoter [43]. Similar to observations by Pollard and colleagues [41], macrophage Ets 2 ablation in the PyMT model did not result in a significant difference in primary tumor burden. However, there was a significant decrease in both the number and size of mammary tumor cell metastatic lesions to the lung [44].

Cancer metastasis to secondary sites involves several stages, including intravasation, homing, extravasation and colonization at the secondary site [45]. To determine if macrophage Ets 2 has an important function at the metastatic lung site, we performed tail-vein injection experiments using a highly metastatic mouse mammary tumor cell line (MET1) [46]. Recipient mice lacking Ets 2 in macrophages had fewer and smaller metastatic lesions growing in the lungs compared to their wildtype littermates, suggesting the reduced metastasis phenotype in transgenic models was at least in part due to loss of Ets 2 function in macrophages in the lung [44]. Gene expression profiling performed on isolated TAMs revealed a program of Ets2 regulated gene expression that was also tumor specific, but entirely distinct from the tumor fibroblast targets. As an example, MMP9 expression was not affected in TAMs. Instead, Ets 2 targets were predominantly repressed in TAMs. In particular, approximately 15 well-annotated negative regulators of angiogenesis were suppressed in TAMs, including Thbsl, Thbs2, Timp1 and Timp3. Using a set of 133 human genes corresponding to the Ets 2 TAM profile, including many of these antiangiogenic genes, we were able to retrospectively predict disease free survival in patients from two human breast cancer microarray data sets [44].

The metastatic spread of tumors that are resistant to conventional therapies remains the leading cause of cancer deaths. While the influence of the microenvironment, particularly macrophages, on tumor growth and metastasis has long been recognized, relatively little is known of the gene pathways and mechanisms in macrophages that promote the dispersion and growth of metastatic tumor cells. The results of our mouse model demonstrate that Ets 2 in tumor macrophages functions to promote angiogenesis and growth of lung metastases without significantly affecting primary tumor burden. Thus, Ets 2 represents a metastasis enhancer that functions in a tumor cell non-autonomous fashion to promote metastasis without affecting primary tumor burden, as opposed to genes termed metastasis suppressors that attenuate metastasis without affecting primary tumor growth.

\section{CONCLUSIONS}

Tumor stroma is heterogeneic and complex. Different resident cells and other non-cellular components contribute to cancer progression at various stages and through differing mechanisms. From lessons learned from studying development, ETS factors can act in a temporally regulated, but often redundant manner to affect cell differentiation and development. In contrast, studies in tumor models suggest that targeting single ETS-factors can have profound effects on tumor growth and spread in a cell non-autonomous fashion (Fig. 1). Why Ets 2 is not redundant in the tumor stroma is not entirely clear. Perhaps as is the case for Etv2 in blood vessel development, the explanation is temporal: Ets 2 may act first in a cascade that leads to a stromal fibroblast able to support tumor growth through multiple mechanisms. Another possibility is that tumor development and progression is a process that depends on rapid evolution and thus bypasses the safeguards that ordered, redundant gene action supplies.

An interesting finding is that Ets 2 can act from different cell compartments to regulate different genes that orchestrate tumor growth and metastasis in complementary fashion. In fibroblasts, Ets2 regulates pro-angiogenic genes, while in macrophages it represses anti-angiogenic genes. The end effect is to synergistically increase angiogenesis necessary for tumor growth and spread.

However, there are still several key questions that need to be addressed in the future: 


\section{Tumor cells}

\section{Stroma}

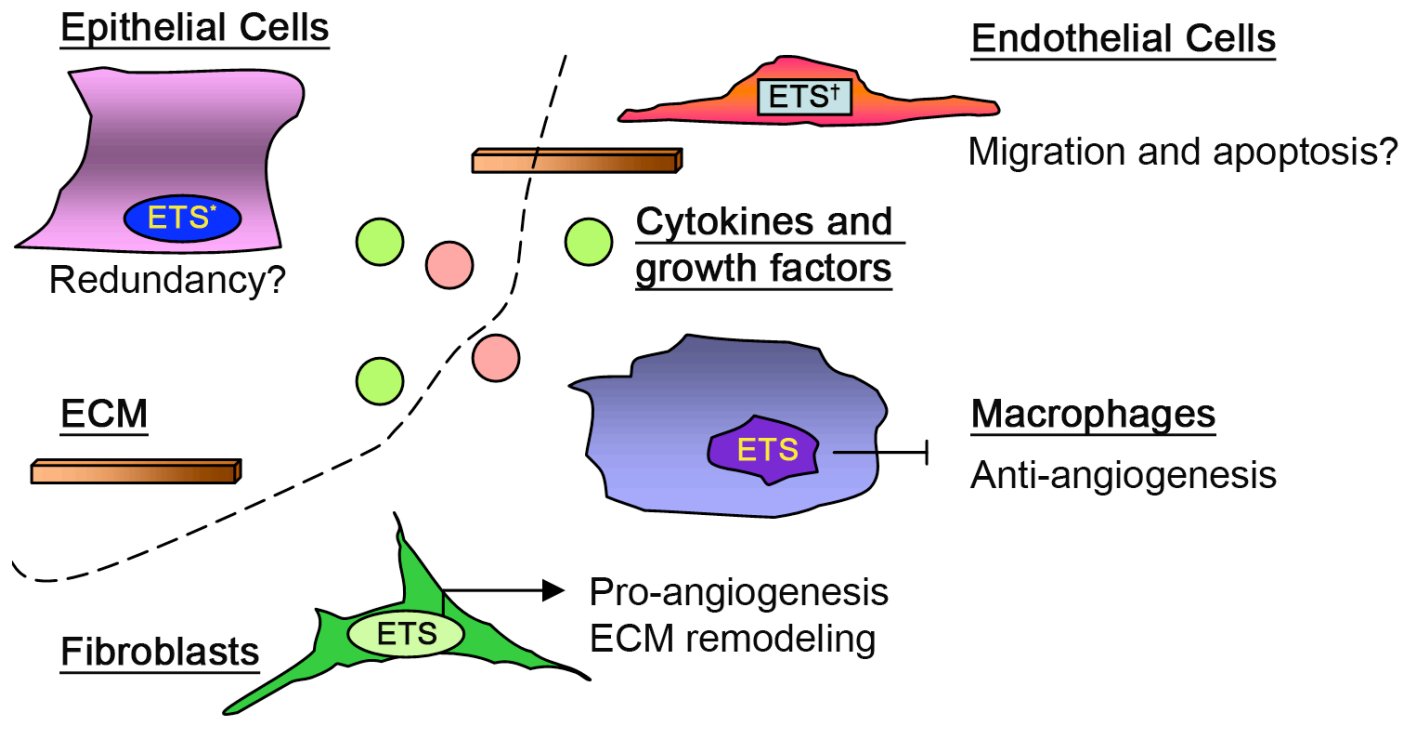

Fig. (1). ETS Functions in Tumor Microenvironment

The tumor microenvironment is comprised of cancerous epithelial cells and stromal cells (i.e. fibroblasts, macrophages and endothelial cells), as well as other non-cellular components including the extracellular matrix (ECM) and cytokines. Strong evidence suggests that ETS factors regulate distinct transcription programs in both fibroblasts and macrophages, which modify the structure and composition of the surrounding ECM. These changes ultimately impact the overall growth of the tumor, with angiogenesis as a key biological processes effected by ETS factors. However, the exact functions of ETS factors in cancer cells and endothelial cells during tumor progression remain to be determined. *, ETS factors may act in a redundant fashion in cancer cells. $\uparrow$, ETS1 and ETS2 are involved in migration and apoptosis of embryonic endothelial cells.

1. What are the roles of ETS factors in tumor endothelial cells? Ets 1 and Ets2 have been shown to regulate endothelial cell survival during embryonic development in a cell-autonomous fashion. Will Ets1 and Ets2 also be redundant during tumor angiogenesis? Will these ETS-factors promote endothelial cell survival in response to cues emanating from these other stromal cell types? Temporal conditional knockout of ETS factors in endothelial cells will provide answers for these questions.

2. What are the functions of other ETS factors in tumor stroma? Are they activated or repressed in tumor stroma? Laser capture microdissection (LCM) coupled with genomic analysis from tumor tissue microarrays has the potential to reveal stromal involvement of these factors. Subsequent genetic model analysis will evaluate their functions during cancer progression.

3. What are the mechanisms responsible for switching the function of ETS2 as activator or repressor in different stromal cell types? What are the cofactors of ETS2 in these cell types? What are the direct targets of ETS factors in tumor associated fibroblasts and macrophages?

4. What are the roles of ETS factors in the microenvironment of other types of cancer besides breast cancer? The genetic studies from Oshima's group and our group focused on mammary tumor models. However, ETS factors are deregulated in other types of cancer as well, including liver, lung and colon cancer. Interestingly, overexpression of Ets 2 appears to protect against tumor progression in a colon cancer mouse model of Down syndrome [47]. Is this inhibitory effect of ETS2 in colon cancer arising from the stroma?

\section{ACKNOWLEDGEMENTS}

We like to thank members of Ostrowski laboratory for helpful discussions. F.L is a recipient of Department of Defense Pre-doctoral Fellowship (W81XWH-08-1-0773). Research in the MCO laboratory is supported by funding from National Institute of Health (P01CA097189 and R01CA053271), Susan Komen foundation and the Evelyn Simmers Foundation.

\section{REFERENCES}

[1] Beug H, von Kirchbach A, Doderlein G, Conscience JF, Graf T. Chicken hematopoietic cells transformed by seven strains of defective avian leukemia viruses display three distinct phenotypes of differentiation. Cell 1979; 18: 375-90.

[2] Leprince D, Gegonne A, Coll J, et al. A putative second cellderived oncogene of the avian leukaemia retrovirus E26. Nature 1983; 306: 395-7.

[3] Gutierrez-Hartmann A, Duval DL, Bradford AP. ETS transcrip-tion factors in endocrine systems. Trends Endocrinol Metab 2007; 18: 150-8.

[4] Seth A, Watson DK, Blair DG, Papas TS. c-ets-2 protooncogene has mitogenic and oncogenic activity. Proc Natl Acad Sci USA 1989; 86: 7833-7.

[5] Seth A, Watson DK. ETS transcription factors and their emerging roles in human cancer. Eur J Cancer 2005; 41: 2462-78. 
[6] Clark JP, Cooper CS. ETS gene fusions in prostate cancer. Nat Rev Urol 2009; 6: 429-39.

[7] Hsu T, Trojanowska M, Watson DK. ETS proteins in biological control and cancer. J Cell Biochem 2004; 91: 896-903.

[8] Hanahan D, Weinberg RA. The hallmarks of cancer. Cell 2000; 100: 57-70.

[9] Powers $\mathrm{S}, \mathrm{Mu} \mathrm{D}$. Genetic similarities between organogenesis and tumorigenesis of the lung. Cell Cycle 2008; 7: 200-4.

[10] Zhang Z, Verheyden JM, Hassell JA, Sun X. FGF-regulated Etv genes are essential for repressing Shh expression in mouse limb buds. Dev Cell 2009; 16: 607-13.

[11] Mao J, McGlinn E, Huang P, Tabin CJ, McMahon AP. Fgfdependent Etv $4 / 5$ activity is required for posterior restriction of Sonic Hedgehog and promoting outgrowth of the vertebrate limb. Dev Cell 2009; 16: 600-6.

[12] Patan S. Vasculogenesis and angiogenesis as mechanisms of vascular network formation, growth and remodeling. J Neurooncol 2000; 50: 1-15.

[13] Dejana E, Taddei A, Randi AM. Foxs and Ets in the transcriptional regulation of endothelial cell differentiation and angiogenesis. Biochim Biophys Acta 2007; 1775: 298-312.

[14] De Val S, Black BL. Transcriptional control of endothelial cell development. Dev Cell 2009; 16: 180-95.

[15] Liu F, Patient R. Genome-wide analysis of the zebrafish ETS family identifies three genes required for hemangioblast differentiation or angiogenesis. Circ Res 2008; 103: 1147-54.

[16] Pham VN, Lawson ND, Mugford JW, et al. Combinatorial function of ETS transcription factors in the developing vasculature. Dev Biol 2007; 303: 772-83.

[17] Lee D, Park C, Lee H, et al. ER71 acts downstream of BMP, Notch, and Wnt signaling in blood and vessel progenitor specification. Cell Stem Cell 2008; 2: 497-507.

[18] Sumanas S, Gomez G, Zhao Y, Park C, Choi K, Lin S. Interplay among Etsrp/ER71, Scl, and Alk8 signaling controls endothelial and myeloid cell formation. Blood 2008; 111: 4500-10.

[19] De Val S, Anderson JP, Heidt AB, Khiem D, Xu SM, Black BL. Mef2c is activated directly by Ets transcription factors through an evolutionarily conserved endothelial cell-specific enhancer. Dev Biol 2004; 275: 424-34.

[20] Ferdous A, Caprioli A, Iacovino M, et al. Nkx2-5 transactivates the Ets-related protein 71 gene and specifies an endothelial/ endocardial fate in the developing embryo. Proc Natl Acad Sci USA 2009; 106: 814-9.

[21] Barton K, Muthusamy N, Fischer C, et al. The Ets-1 transcription factor is required for the development of natural killer cells in mice. Immunity 1998; 9: 555-63.

[22] Loughran SJ, Kruse EA, Hacking DF, et al. The transcription factor Erg is essential for definitive hematopoiesis and the function of adult hematopoietic stem cells. Nat Immunol 2008; 9: 810-9.

[23] Bories JC, Willerford DM, Grévin D, et al. Increased T-cell apoptosis and terminal B-cell differentiation induced by inactivation of the Ets-1 proto-oncogene. Nature 1995; 377: 635-8.

[24] Spyropoulos DD, Pharr PN, Lavenburg KR, et al. Hemorrhage, impaired hematopoiesis, and lethality in mouse embryos carrying a targeted disruption of the Fli1 transcription factor. Mol Cell Biol 2000; 20: 5643-52.

[25] Hart A, Melet F, Grossfeld P, et al. Fli-1 is required for murine vascular and megakaryocytic development and is hemizygously deleted in patients with thrombocytopenia. Immunity 2000; 13: 167-77.
[26] Kruse EA, Loughran SJ, Baldwin TM, et al. Dual requirement for the ETS transcription factors Fli-1 and Erg in hematopoietic stem cells and the megakaryocyte lineage. Proc Natl Acad Sci USA 2009; 106: 13814-9.

[27] Wei G, Srinivasan R, Cantemir-Stone CZ, et al. Ets1 and Ets2 are required for endothelial cell survival during embryonic angiogenesis. Blood 2009; 114: 1123-30.

[28] Lettice LA, Hill RE. Preaxial polydactyly: a model for defective long-range regulation in congenital abnormalities. Curr Opin Genet Dev 2005; 15: 294-300.

[29] Sementchenko VI, Watson DK. Ets target genes: past, present and future. Oncogene 2000; 19: 6533-48.

[30] Man A, Young L, Tynan J, et al. Ets2-dependent stromal regulation of mouse mammary tumors. Mol Cell Biol 2003; 23: 8614-25.

[31] Tynan JA, Wen F, Muller WJ, Oshima RG. Ets2-dependent microenvironmental support of mouse mammary tumors. Oncogene 2005; 24: 6870-6.

[32] Kalluri R, Zeisberg M. Fibroblasts in cancer. Nat Rev Cancer 2006; 6: 392-401.

[33] Bhowmick NA, Chytil A, Plieth D, et al. TGF-beta signaling in fibroblasts modulates the oncogenic potential of adjacent epithelia. Science 2004; 303: 848-51.

[34] Trimboli AJ, Cantemir-Stone CZ, Li F, et al. Pten in stromal fibroblasts suppresses mammary epithelial tumours. Nature 2009; 461: 1084-91.

[35] Overall CM, Kleifeld O. Tumour microenvironment - opinion: validating matrix metalloproteinases as drug targets and anti-targets for cancer therapy. Nat Rev Cancer 2006; 6: 227-39.

[36] Bergers G, Brekken R, McMahon G, et al. Matrix metalloproteinase-9 triggers the angiogenic switch during carcinogenesis. Nat Cell Biol 2000; 2: 737-44.

[37] Pollard JW. Trophic macrophages in development and disease. Nat Rev Immunol 2009; 9: 259-70.

[38] Joyce JA, Pollard JW. Microenvironmental regulation of metastasis. Nat Rev Cancer 2009; 9: 239-52.

[39] Condeelis J, Pollard JW. Macrophages: obligate partners for tumor cell migration, invasion, and metastasis. Cell 2006; 124: 263-6.

[40] Sica A, Larghi P, Mancino A, et al. Macrophage polarization in tumour progression. Semin Cancer Biol 2008; 18: 349-55.

[41] Lin EY, Nguyen AV, Russell RG, Pollard JW. Colony-stimu-lating factor 1 promotes progression of mammary tumors to malignancy. J Exp Med 2001; 193: 727-40.

[42] Fowles LF, Martin ML, Nelsen L, et al. Persistent activation of mitogen-activated protein kinases p42 and p44 and Ets-2 phosphorylation in response to colony-stimulating factor $1 / \mathrm{c}-\mathrm{fms}$ signaling. Mol Cell Biol 1998; 18: 5148-56.

[43] Clausen BE, Burkhardt C, Reith W, Renkawitz R, Forster I. Conditional gene targeting in macrophages and granulocytes using LysMcre mice. Transgenic Res 1999; 8: 265-77.

[44] Zabuawala T, Taffany DA, Sharma SM, et al. An Ets2-driven transcriptional program in tumor-associated macrophages promotes tumor metastasis. Cancer Res 2010; 70: 1323-33.

[45] Gupta GP, Massague J. Cancer metastasis: building a framework. Cell 2006; 127: 679-95.

[46] Borowsky AD, Namba R, Young LJ, et al. Syngeneic mouse mammary carcinoma cell lines: two closely related cell lines with divergent metastatic behavior. Clin Exp Metastasis 2005; 22: 4759.

[47] Sussan TE, Yang A, Li F, Ostrowski MC, Reeves RH. Trisomy represses Apc(Min)-mediated tumours in mouse models of Down's syndrome. Nature 2008; 451: 73-5. 